Provided for non-commercial research and education use. Not for reproduction, distribution or commercial use.

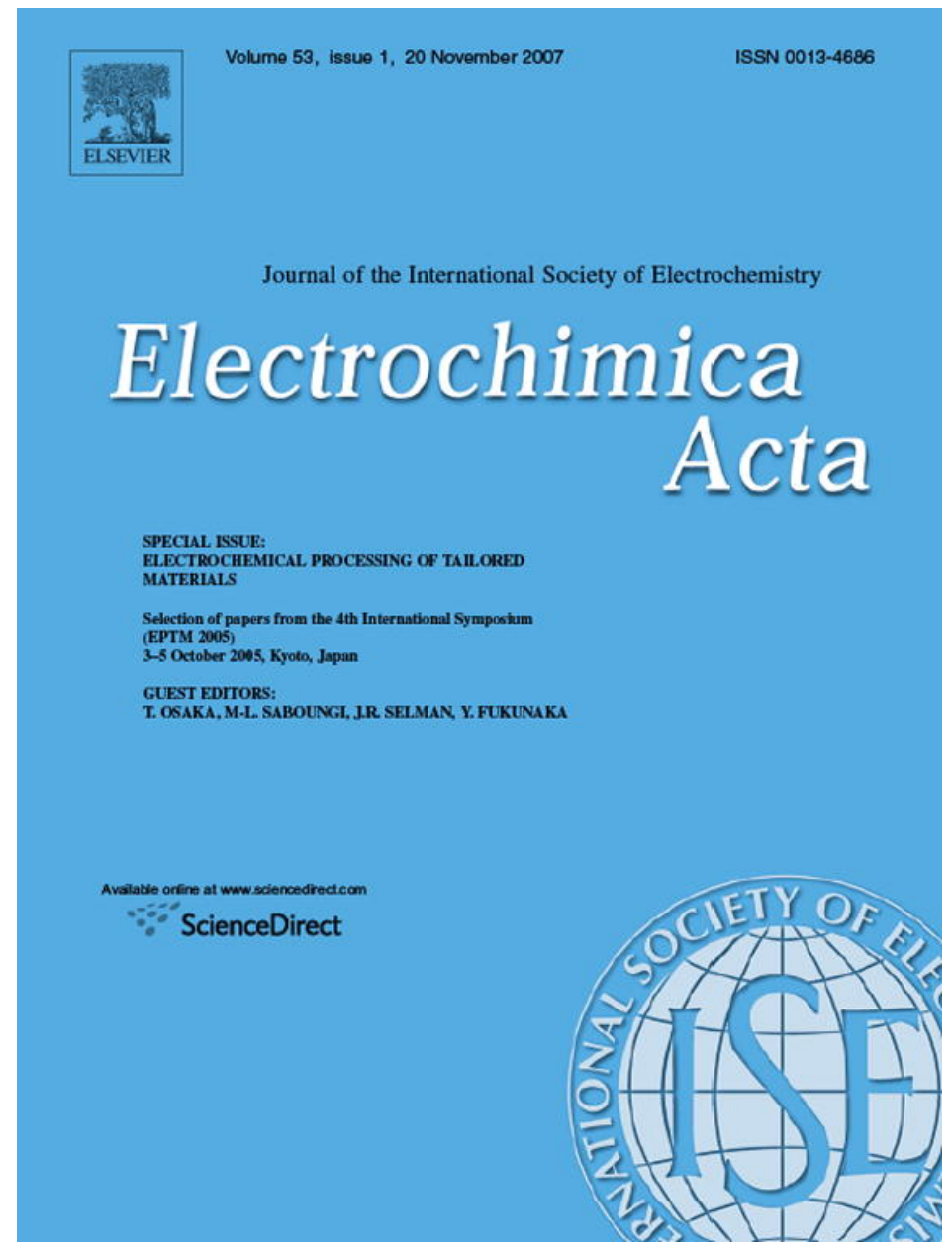

This article was published in an Elsevier journal. The attached copy

is furnished to the author for non-commercial research and education use, including for instruction at the author's institution, sharing with colleagues and providing to institution administration.

Other uses, including reproduction and distribution, or selling or licensing copies, or posting to personal, institutional or third party websites are prohibited.

In most cases authors are permitted to post their version of the article (e.g. in Word or Tex form) to their personal website or institutional repository. Authors requiring further information regarding Elsevier's archiving and manuscript policies are encouraged to visit:

http://www.elsevier.com/copyright 


\title{
Curvature enhanced adsorbate coverage mechanism for bottom-up superfilling and bump control in damascene processing
}

\author{
T.P. Moffat*, D. Wheeler, S.-K. Kim, D. Josell \\ Materials Science and Engineering Laboratory, National Institute of Standards and Technology, \\ Gaithersburg, MD 20899, United States \\ Received 1 December 2005; received in revised form 2 March 2007; accepted 2 March 2007 \\ Available online 12 March 2007
}

\begin{abstract}
Superconformal electrodeposition is explained by the curvature enhanced adsorbate coverage (CEAC) mechanism. A CEAC model is used to quantitatively explain shape evolution during bottom-up "superfill" of trenches and vias. The bottom-up filling dynamic is seen to be a consequence of competitive adsorption between a rate suppressing polyethylene glycol-chloride layer (PEG-Cl) and a depassivating or rate accelerating species comprised of sulfonate-terminated propane thiol/disulfide and chloride (SPS-Cl). The SPS is the stronger surfactant and it adsorbs concurrently with displacement of the more rapidly formed PEG-Cl layer; growth on non-planar geometries thus leads to enrichment of SPS on advancing concave surfaces and dilution on convex sections. Because the metal deposition rate rises monotonically with the local accelerator (SPS-Cl) surface coverage, this yields positive feedback that naturally gives rise to desirable bottom-up superfilling. As the accelerating action of SPS can also be quenched by the addition of a cationic surfactant (LEV), additive concentrations can be optimized to allow SPS derived bottom-up superfilling followed by LEV induced SPS deactivation. The LEV addition can thus be used to inhibit overshoot that otherwise would result in undesirable bump formation above the features. This paper presents a summary of electroanalytical experiments and simulations designed to determine the mechanism and rate constants of the adsorbate interactions during copper deposition in the PEG-Cl-SPS-LEV system as well as predictions of feature filling using the CEAC shape change model.
\end{abstract}

(C) 2007 Elsevier Ltd. All rights reserved.

Keywords: ULSI; Damascene and additives; MEMS

\section{Introduction}

State of the art manufacturing of semiconductor devices involves electrodepositon of copper for device wiring [1]. Current practice depends on the use of electrolyte additives that affect the local deposition rate to achieve superconformal, bottom-up filling of trenches and vias [2]. Studies of three-component additive packages (accelerator, halide and suppressor) and four-component additive packages (accelerator, halide, suppressor and leveler) have provided much information on the filling process [3-20]. In particular, the curvature enhanced accelerator coverage (CEAC) mechanism was developed to describe the effect of area change on adsorbate coverage and explore the consequences on the rate of metal deposi-

\footnotetext{
* Corresponding author.

E-mail address: thomas.moffat@nist.gov (T.P. Moffat).
}

tion and resulting shape evolution during growth on non-planar substrates. As such, CEAC-based models have been shown to quantitatively describe the superconformal film growth that is responsible for "bottom-up filling" of sub-micrometer features in damascene processing [13-16]. CEAC-based models have also provided an explanation for the beneficial effects of certain additives known as "brighteners" on roughness evolution $[21,22]$. The generality of the CEAC mechanism has been demonstrated by successful application to quantify superfilling of sub-micrometer features during silver [23] and gold [24] electrodeposition and copper chemical vapor deposition [25]. Most recently, the CEAC mechanism has been extended further to explain the effect of an additional constituent, often referred to as a leveler, that is used to attenuate the undesired overfill phenomenon [26].

The main characteristic of three-component $\mathrm{Cu}$ superfilling electrolytes is competition between inhibiting and accelerating species for electrode surface sites [2-20]. A model "three 
component" additive package for copper superfilling contains a mixture $1 \mathrm{mmol} / \mathrm{L}$ chloride, a $\mu \mathrm{mol} / \mathrm{L}$ concentration of accelerator such as SPS $\left(\mathrm{Na}_{2}\left(\mathrm{SO}_{3}\left(\mathrm{CH}_{2}\right)_{3} \mathrm{~S}\right)_{2}\right)$ and an inhibitor, such as PEG (polyethylene glycol, $M_{\mathrm{W}} \sim 1000-20,000$ ). The surfactant nature of the inhibitor can lead to rapid formation of a thin passivating PEG-based film on the electrode during its immersion through the air-electrolyte interface. Subsequently, the passivating layer is disrupted by the adsorption of the catalytic sulfonate-terminated short chain thiol/disulfide (i.e. SPS); the thiol/disulfide head group tethers the catalyst to the surface while the sulfonate terminal group interacts to prevent reformation of the PEG blocking layer [2,6]. This displacement of adsorbed PEG by SPS, an exponential function of potential, impacts the metal deposition rate through a monotonic dependence on catalyst coverage $[2,6]$. Under certain conditions this activation of the metal deposition rate is sustained by the tendency of the catalyst to remain segregated at the growing interface $[2,6,9,10]$. The concurrent accumulation and segregation of adsorbed catalyst results in hysteretic voltammetric curves [2,6,8,15,18,19], rising chronoamperometric transients $[2,6,8]$ and depolarizing chronopotentiometry $[8,20]$. In contrast, addition of a suitable cationic surfactant (LEV) to the PEG-Cl-SPS system results in deactivation of the SPS [26-28]. This is ascribed to an ion pairing interaction between the cationic head group of LEV and the sulfonate tail group of SPS $[26,28]$ that decreases the deposition rate on the SPS catalyzed surface to values similar to that observed on a PEG-Cl passivated surface [28]. This deactivation is manifest as an attenuation of the voltammetric hysteresis, rising current transients or depolarizing chronopotentiometry. Thus standard electroanalytical methods can be used to quantify the competitive adsorption process in multi-additive systems by examining the impact of adsorbates on the kinetics of metal deposition.

The CEAC construct combines the competitive adsorption dynamics obtained from the studies on planar substrates with an adsorbate conservation requirement upon area change to enable predictions of morphological evolution during deposition on nonplanar substrates [2]. In the PEG-Cl-SPS case, the coverage of the more strongly tethered SPS-Cl catalytic species increases on advancing concave sections and decreases on advancing convex segments. Since the deposition rate rises monotonically with catalyst coverage, this naturally gives rise to bottom-up filling of features and, more generally, superconformal film growth. The area change effect becomes increasingly important as the dimensions of the surface profile shrink into the sub-micrometer regime where effects arising from lateral compositional gradients within the electrolyte must necessarily become less significant. To date, three different shape change CEAC algorithms of varying complexity have been used to describe the filling of sub-micrometer features such as trenches and vias [2,13-15,29]. The models successfully predict interface evolution from the initial incubation period of (near) conformal growth through superconformal bottom-up filling to the bump formation over filled features known as "momentum plating". More recently, it was recognized that area change effects also play an important role in the SPS deactivation process associated with the adsorption of cationic "leveling" surfactants (LEV) used in four component systems to control momentum plating [26].

This report gives an overview of the use of electroanalytical measurements on planar electrodes for quantifying competitive adsorption and its effect on $\mathrm{Cu}$ deposition kinetics in a $1.8 \mathrm{~mol} / \mathrm{L}$ $\mathrm{H}_{2} \mathrm{SO}_{4}-0.25 \mathrm{~mol} / \mathrm{L} \mathrm{CuSO}_{4}$ electrolyte containing PEG-Cl-SPS or PEG-Cl-SPS-LEV additive combinations. The experimentally derived kinetics parameters are then combined with the adsorbate conservation stipulations of the CEAC mechanism to predict shape evolution during copper deposition in a submicrometer trench.

\section{Competitive adsorption model}

\subsection{Growth equation}

The copper deposition rate for the SPS-Cl-PEG-LEV system is taken as the sum of the surface-fraction $\theta_{j}$ weighted deposition rates or current density $i_{j}$ for metal deposition on bare and/or fully modified surfaces: $i_{\mathrm{PEG}}^{0}$ is the exchange current density for the PEG suppressed surface $\left(\theta_{\mathrm{PEG}}=1\right), i_{\text {SPS }}^{0}$ for SPS accelerator covered surface $\left(\theta_{\mathrm{SPS}}=1\right), i_{\mathrm{LEV}}^{0}$ for leveler covered surface $\left(\theta_{\mathrm{LEV}}=1\right)$ and $i_{\mathrm{FREE}}$ for bare surface $\left(\theta_{\mathrm{FREE}}=1\right)$. The metal deposition rate is also taken to be proportional to the local interfacial metal ion concentration $C_{\mathrm{m}}$ so that the current density is written as

$$
\begin{aligned}
& i\left(\theta_{\mathrm{PEG}}, \theta_{\mathrm{SPS}}, \theta_{\mathrm{LEV}}, \theta_{\mathrm{FREE}}, \eta, C\right) \\
& \quad=\frac{C_{\mathrm{m}}}{C_{\mathrm{m}}^{0}} \sum_{j} \theta_{j} i_{j}^{0}\left(\exp \left(-\frac{\alpha_{j} f}{R T} \eta\right)-\exp \left(\frac{\left(1-\alpha_{j}\right) F}{R T} \eta\right)\right)
\end{aligned}
$$

with $j=$ PEG, SPS, LEV and FREE, Faraday's constant $F=96,485 \mathrm{C} / \mathrm{mol}$ and Boltzmann's constant $R=8.314 \mathrm{~J} /$ $(\mathrm{mol} \mathrm{K})$. Chloride is a required co-adsorbate for both the suppressor and accelerator, so the coverages should strictly be $\theta_{\text {PEG-Cl }}$ and $\theta_{\text {SPS-Cl }}$. However, since halide is plentiful in the electrolyte the needs of the respective co-adsorbates are readily met and thus chloride coverage is not explicitly tracked. The deposition rate is expressed as an interface velocity $v$ normal to the surface using Faraday's law $v=i \Omega / n F$ with $\Omega$ the molar volume of the deposited metal and $n$ the charge of the metal ion in the electrolyte.

For the purposes of this paper electroanalytical and feature filling experiments were simulated assuming that the initial immersed surface was rapidly covered with PEG-Cl $\left(\theta_{\mathrm{PEG}}=1\right.$ at $t=0)$ and that any free-sites that subsequently develop on the advancing convex surfaces were rapidly, i.e. instantaneously, covered with PEG-Cl (i.e. $\theta_{\mathrm{FREE}}=0$ ). These approximations are rationalized by the exposure of the electrode to the PEG enriched air-electrolyte interface during immersion prior to the onset of plating combined with the high PEG/SPS concentration ratio over most of the SPS range examined. These stipulations enable attention to be focused on the subsequent evolution of $\theta_{\text {SPS }}$ and $\theta_{\mathrm{LEV}}$; the limitations of the approximations will be explored elsewhere. 


\subsection{SPS and LEV coverage evolution equations}

Mass conservation yields,

$$
\begin{aligned}
\frac{\mathrm{d} \theta_{\mathrm{SPS}}}{\mathrm{d} t}= & \kappa v \theta_{\mathrm{SPS}}+C_{\mathrm{SPS}} k_{\mathrm{SPS}}^{+}\left(1-\theta_{\mathrm{SPS}}-\theta_{\mathrm{LEV}}\right) \\
& -\theta_{\mathrm{SPS}} C_{\mathrm{LEV}} k_{\mathrm{LEV}}^{+}-k_{\mathrm{SPS}}^{-}\left(\theta_{\mathrm{SPS}}\right)^{q}
\end{aligned}
$$

for the surface coverage of adsorbed accelerator, and

$$
\frac{d \theta_{\mathrm{LEV}}}{\mathrm{d} t}=\kappa v \theta_{\mathrm{LEV}}+C_{\mathrm{LEV}} k_{\mathrm{LEV}}^{+}\left(1-\theta_{\mathrm{LEV}}\right)-k_{\mathrm{L}}^{-} \theta_{\mathrm{LEV}}
$$

for the leveler coverage. The first term on the right side of the evolution Eqs. (2) and (3) accounts for the impact of area change on the local coverage of the given adsorbate; this fundamental term of the CEAC formalism ensures mass conservation for an adsorbate that remains segregated on a surface of curvature $\kappa$ during metal deposition at velocity $v$. Positive $\kappa$ corresponds to a concave interface such as the bottom of a trench while $\kappa=0$ defines a planar surface. The second term in each equation accounts for adsorbate accumulation from the electrolyte; $C_{i}$ is the concentration of the given additive adjacent to the metal/electrolyte interface and $k_{i}^{+}$equals the adsorption rate constant. The respective accumulation terms reflect the proposed hierarchy of surface binding interactions; the accelerator is only able to adsorb on sites occupied by suppressor (i.e., by displacement) while the leveler is capable of adsorbing on sites occupied by either the suppressor or accelerator. The rate constant for SPS adsorption, $k_{\text {SPS }}^{+}$is potential dependent, the rate increasing exponentially with overpotential $\eta$ according to

$k_{\mathrm{ads}}=k_{0} \exp \left(-\frac{\alpha_{\mathrm{ads}} F \eta}{R T}\right)$

The symmetry of the activation barrier, $\alpha_{\text {ads }}$, and the preexponential term, $k_{0 S P S}$, have been determined by fitting the experimental $\eta-i$ curves on the planar substrates over the range of SPS concentrations in the PEG-Cl-SPS system [2]. The apparent rate constant convolves SPS adsorption with PEG desorption (i.e. $\theta_{\mathrm{SPS}}+\theta_{\mathrm{PEG}}=1$, thus for SPS to occupy a PEG covered site, desorption of PEG must first occur, the respective desorption and adsorption steps may exhibit distinct potential dependences that are combined in Eq. (4)). In contrast, in the absence of data suggesting otherwise, a single potential-independent rate constant, $k_{\mathrm{LEV}}^{+}$, is used to describe the leveler-PEG and leveler-SPS displacement process in the PEG-Cl-SPS-LEV system.

The third term in the accelerator evolution Eq. (2) represents deactivation of accelerator by adsorbing leveler; it equals the rate at which leveler accumulates from the electrolyte weighted by the fraction of sites where it displaces or deactivates adsorbed SPS accelerator. Physically, the interaction could involve phenomena as disparate as masking accelerator activity by forming an ion pair on the surface or displacing accelerator into the electrolyte or into the growing solid (i.e., incorporation). The latter is consistent with recent experimental findings of a monotonic increase in accelerator incorporation in the solid with increasing leveler concentration [30]. The final term in the evolution Eqs. (2) and (3) describes the independent deactivation of the respective adsorbate species, possibly by incorporation into the growing solid or desorption of the molecule or some fragment back into the electrolyte. For the accelerator deactiviation (in Eq. (2)) an empirical form previously derived from chronoamperometric measurements of deactivation of SPS-derivitized electrodes is used [2]. The deactivation process was observed to be potential dependent, passing through a maximum ( $\left.V_{\text {peak }}\right)$ near $-0.095 \mathrm{~V}$, the potential dependence being described by an asymmetric Gaussian function with constants, $A_{1}, A_{2}, A_{3}$ and $A_{4}$ :

$$
k_{\mathrm{SPS}}^{-}=A_{1}+\frac{A_{2}}{\exp \left(-A_{3}\left(\eta-V_{\text {peak }}\right)\right)+\exp \left(A_{4}\left(\eta-V_{\text {peak }}\right)\right)}
$$

The expression for leveler deactivation in Eq. (3) invokes a simple, potential-independent rate constant that is formally indistinguishable from Langmuir desorption. Thus, deactivation is first order in leveler coverage and involves either desorption or alternatively consumption and incorporation into the solid.

Importantly, the first term in Eqs. (2) and (3), respectively, do not contribute to additive evolution during deposition on smooth planar electrodes; this allows conventional electroanalytical measurements on such electrodes to be used to quantify all other terms in Eqs. (1)-(3). In contrast, during deposition on non-planar, e.g. lithographically patterned surfaces, the area change terms can dominate the local response of a surface. For the two component accelerator-suppressor systems, the localized increase of accelerator coverage at the bottoms of features that arises from this term is responsible for the bottom-up filling behavior desired for damascene metallization [2,13-15]. Significantly, an important variation can occur in the presence of leveler. Specifically, Eqs. (2) and (3) apply only as long as there is still suppressor that can be removed from the surface (i.e., $\left.\theta_{\mathrm{SPS}}+\theta_{\mathrm{LEV}}<1\right)$. If the surface is fully covered with adsorbed accelerator and leveler (i.e., $\theta_{\mathrm{SPS}}+\theta_{\mathrm{LEV}}=1$ ) and Eqs. (2) and (3) yield

$\frac{\mathrm{d} \theta_{\mathrm{SPS}}}{\mathrm{d} t}+\frac{\mathrm{d} \theta_{\mathrm{LEV}}}{\mathrm{d} t}>0$

then $\theta_{\text {LEV }}$ continues to evolve according to Eq. (3), but the $\theta_{\text {SPS }}$ must now accommodate the evolution of the leveler according to

$\frac{\mathrm{d} \theta_{\mathrm{SPS}}}{\mathrm{d} t}=\frac{\mathrm{d} \theta_{\mathrm{LEV}}}{\mathrm{d} t}$

It is through Eq. (7) that adsorbed accelerator is deactivated by adsorbed leveler during area reduction in suppressor-free regions. Note this inverse of the curvature enhanced accelerator coverage process is entirely distinct from the classical picture of leveling known as diffusion-adsorption-consumption models [31-37]. As with early CEAC models, area change effects can dominate over coverage differentiation arising from boundary layer transport controlled process. This naturally follows from the relevant length scales involved, i.e. coverage changes due to rapid area change within sub-micrometer $(\sim 100 \mathrm{~nm})$ features overwhelm any lateral variations associated with diffusional fluxes over the hydrodynamic boundary layer $(10-100 \mu \mathrm{m})$. In the shape change simulations that follow, locations where the 
accelerator coverage saturates, i.e. $\theta_{\text {SPS }}=1$ and Eq. (2) yields $\mathrm{d} \theta_{\text {SPS }} / \mathrm{d} t>0$, then $\theta_{\text {SPS }}=1$ was imposed and any excess accelerator was modeled as incorporated into the growing solid; the same was done for adsorbed leveler. Though not done here, it is straightforward to model desorption of either species into the electrolyte with appropriate modification of the mass conservation boundary conditions at the interface.

In addition to adsorption kinetics and their effect on the metal deposition rate, depletion effects associated with limited transport of the reactants must be fully considered. In the general case, such as the trench filling simulations presented in this work, the full time-dependent diffusion equations for transport of $\mathrm{Cu}^{2+}$, SPS and LEV within the evolving boundary layer and unfilled region of the trench are evaluated using

$\frac{\partial C_{j}}{\partial t}=D_{j} \nabla^{2} C_{j}$

with uniform bulk concentrations imposed at the start of deposition $(t=0)$. The remaining boundary conditions are obtained by imposing mass conservation along the growth surface. For the metal this yields

$D_{\mathrm{M}} \nabla C_{\mathrm{M}} \cdot \hat{n}=v\left(\frac{1}{\Omega}\right)$

which equates metal deposited on the surface with that arriving from the electrolyte. Conservation of the additives at the growth surface is enforced by equating the rate of arrival from the electrolyte with the rate of adsorption on the surface according to

$$
\begin{aligned}
& D_{\mathrm{SPS}} \nabla C_{\mathrm{SPS}} \cdot \hat{n}=\Gamma_{0}^{\mathrm{SPS}} C_{\mathrm{SPS}} k_{\mathrm{SPS}}^{+}\left(1-\theta_{\mathrm{SPS}}-\theta_{\mathrm{LEV}}\right), \\
& D_{\mathrm{LEV}} \nabla C_{\mathrm{LEV}} \cdot \hat{n}=\Gamma_{0}^{\mathrm{LEV}} C_{\mathrm{LEV}} k_{\mathrm{LEV}}^{+}\left(1-\theta_{\mathrm{LEV}}\right)
\end{aligned}
$$

where $\hat{n}$ is the local unit normal of the surface (pointing into the electrolyte). Implicit in Eq. (10) is the assumption that adsorbates eliminated through consumption are buried (or deactivated), as was imposed during area reduction of saturated surfaces, so that they do not appear in the mass balance; equation modification where this is not the case is straightforward. In the case of slow interface kinetics and negligible leveler depletion effects, these equations are also consistent with Langmuir desorption. Experiments, such as to assess contaminants in deposits, are required to determine the actual underlying mechanism of adsorbate elimination.

For steady-state electrochemical measurements on planar electrodes either natural convection associated with $\mathrm{Cu}^{2+}$ depletion gradients or enforced hydrodynamics may be used to establish the boundary layer approximation. The interface concentrations of $\mathrm{Cu}^{2+}$, SPS and the LEV species are then defined by mass balance according to

$$
\begin{aligned}
& C_{\mathrm{M}}=C_{\mathrm{M}}^{\infty}-\frac{\delta i}{2 D} \\
& C_{\mathrm{SPS}}=\frac{C_{\mathrm{SPS}}^{\text {bulk }}}{1+\left(k_{\mathrm{SPS}}^{+} \Gamma_{0}^{\mathrm{SPS}} \delta\left(1-\theta_{\mathrm{SPS}}-\theta_{\mathrm{LEV}}\right)\right) / D_{\mathrm{SPS}}}
\end{aligned}
$$

\begin{tabular}{|c|c|}
\hline Parameter & Value \\
\hline$\alpha_{\mathrm{PEG}}\left(\right.$ and $\left.\alpha_{\mathrm{LEV}}\right)$ & 0.5 \\
\hline$\alpha_{\mathrm{SPS}}$ & 0.4 \\
\hline$i_{\mathrm{PEG}}^{0}\left(\right.$ and $\left.i_{\mathrm{LEV}}^{0}\right)$ & $0.03 \mathrm{~mA} / \mathrm{cm}^{2}$ \\
\hline$i_{\mathrm{SPS}}^{0}$ & $\begin{array}{l}4.5 \mathrm{~mA} / \mathrm{cm}^{2} \\
298 \mathrm{~K}\end{array}$ \\
\hline$\eta$ (fixed for feature filling) & $-0.25 \mathrm{~V}$ \\
\hline$C_{\mathrm{m}}^{0}$ & $2.4 \times 10^{-4} \mathrm{~mol} / \mathrm{cm}^{3}$ \\
\hline$C_{\mathrm{SPS}}^{\text {bulk }}$ & $x \mathrm{~mol} / \mathrm{cm}^{3}$ \\
\hline $\mathrm{C}_{\mathrm{LEV}}^{\text {bulk }}$ & $x \mathrm{~mol} / \mathrm{cm}^{3}$ \\
\hline$D_{\mathrm{m}}$ & $4 \times 10^{-6} \mathrm{~cm}^{2} / \mathrm{s}$ \\
\hline$D_{\mathrm{SPS}}\left(\right.$ and $\left.D_{\mathrm{LEV}}\right)$ & $4 \times 10^{-6} \mathrm{~cm}^{2} / \mathrm{s}$ \\
\hline$\delta$ & $9.87 \times 10^{-3} \mathrm{~cm}$ \\
\hline$\Omega$ & $7.1 \mathrm{~cm}^{3} / \mathrm{mol}$ \\
\hline$n$ & 2 \\
\hline$\Gamma_{0}^{\mathrm{SPS}}\left(\right.$ and $\left.\Gamma_{0}^{\mathrm{LEV}}\right)$ & $6.35 \times 10^{-10} \mathrm{~mol} / \mathrm{cm}^{2}$ \\
\hline$k_{\mathrm{SPS}}^{+}$ & $f(\eta)$ see Eq. $(3)$ \\
\hline$\alpha_{\text {ads }}$ & 0.6 \\
\hline$k_{0}$ & $260 \mathrm{~cm}^{3} /(\mathrm{mol} \mathrm{s})$ \\
\hline$k_{\mathrm{SPS}}^{-}$ & $f(\eta)$ see Eq. (4) \\
\hline$A_{1}$ & $0.0008 \mathrm{~s}^{-1}$ \\
\hline$A_{2}$ & 0.3 \\
\hline$A_{3}$ & 35 \\
\hline$A_{4}$ & 60 \\
\hline$V_{\text {peak }}$ & $-0.095 \mathrm{~V}$ \\
\hline$q$ & $=m \eta+b, m=4 \mathrm{~V}^{-1}, b=2.65$ \\
\hline$k_{\mathrm{LEV}}^{+}$ & $1.8 \times 10^{4} \mathrm{~cm}^{3} /(\mathrm{mol} \mathrm{s})$ \\
\hline$k_{\mathrm{LEV}}^{-\mathrm{LEV}}$ & $5 \times 10^{-4} \mathrm{~cm}^{-1}$ \\
\hline Trench depth & $4 \times 10^{-5} \mathrm{~cm}$ \\
\hline Width at half trench depth & $2.7 \times 10^{-5} \mathrm{~cm}$ \\
\hline$\phi$ (sidewall tilt) & $5^{\circ}$ \\
\hline
\end{tabular}

Table 1

Kinetic parameters

$C_{\mathrm{LEV}}=\frac{C_{\mathrm{LEV}}^{\mathrm{bulk}}}{1+\left(k_{\mathrm{LEV}}^{+} \Gamma_{0}^{\mathrm{LEV}} \delta\left(1-\theta_{\mathrm{LEV}}\right)\right) / D_{\mathrm{LEV}}}$

Values for the diffusion coefficients $D_{i}$, saturation surface coverages $\Gamma_{0}^{i}$, boundary layer thickness $\delta$ and all the other kinetic parameters derived from prior experiments are summarized in Table 1.

Summarizing, the key CEAC elements relevant to superfilling in the PEG-Cl-SPS systems are (a) displacement of adsorbed PEG from the surface by SPS adsorption with potential dependent kinetics and (b) SPS-induced displacement of PEG from the surface during area reduction that accompanies an advancing concave surface. In the added presence of a leveler two additional processes can occur whereby the leveler deactivates the adsorbed SPS accelerator: (i) adsorption from the electrolyte and (ii) lateral interactions during area reduction of surface segments that are saturated with catalyst and leveler. Eqs. (2) and (3) implicitly stipulate that the leveler can deactivate or displace both SPS and PEG from the surface.

In subsequent modeling shown below it will be assumed that the leveler-saturated surface exhibits the same metal deposition kinetics as the PEG-Cl suppressor-saturated surface; this approximation is supported by recent experimental work on the prototypical cationic surfactant levelers $\left(\mathrm{CH}_{3}\right)_{3} \mathrm{C}_{12} \mathrm{H}_{25} \mathrm{~N}^{+} \mathrm{Cl}^{-}$ (DTAC) [28] and polyethyleneimine (PEI) [38]. An important 
consequence of this simplifying assumption is that interface motion is unaffected by the competition between leveler and PEG for surface sites. In contrast, the impact of adsorbate interactions on the spatially evolving distribution of SPS, coupled with the $\mathrm{Cu}^{2+}$ depletion gradient, accounts for the key aspects of shape evolution during superfilling and bump formation, during practical damascene processing conditions.

\section{Electroanalytical simulations}

\subsection{PEG-Cl-SPS system}

Conventional electroanalytical methods are widely used for both development of plating electrolytes and process control of plating processes. Previous investigations have shown that characteristics such as hysteretic voltammetry, rising chronoamperometric traces and depolarizing chronopotentiometric transients are important indicators of superfilling performance in a variety of chemically distinct systems, e.g., copper [2,6,8,15,18-20], silver [23] and gold [24]. The competitive adsorption model outlined above (and variations thereof) is capable of generating all reported aspects of the above analytical measurements. A brief overview of the simulations results based on kinetics parameters derived from prior experiments $[2,6]$ follows. Those interested in comparison with experimental results are directed to Ref. [2].

An example of voltammetric hysteresis accompanying copper deposition from a PEG-Cl-SPS electrolyte containing $0,0.5$, 5 and $50 \mu \mathrm{mol} / \mathrm{L} \mathrm{SPS}$ is given in Fig. 1; it should be reiterated that, like all the figures presented herein, these are simulations obtained from the equations detailed above using the (experimentally derived) kinetics contained in Table 1 . In the absence of SPS, PEG-Cl forms a blocking layer on the electrode that inhibits the metal deposition reaction by two orders of magnitude relative to the additive-free case; experiments also reveal minor relaxation of the passivating layer convolved with roughness evolution that lead to subtle changes with potential cycling that are not modeled here. The addition of SPS to a PEG-Cl plating bath results in progressive acceleration of the deposition rate

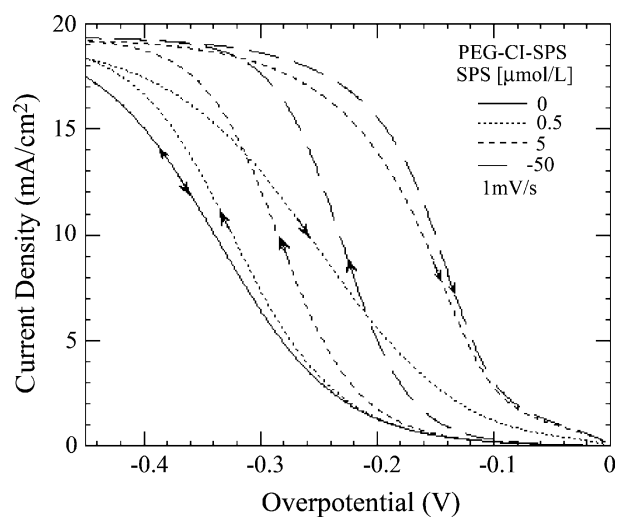

Fig. 1. Slow scan voltammetry reveals the accelerating influence of SPS on the copper deposition rate in an electrolyte comprised of $1.8 \mathrm{~mol} / \mathrm{L}$ $\mathrm{H}_{2} \mathrm{SO}_{4}+0.25 \mathrm{~mol} / \mathrm{L} \quad \mathrm{CuSO}_{4}+88 \mu \mathrm{mol} / \mathrm{L} \quad \mathrm{PEG}+1 \mathrm{mmol} / \mathrm{L} \mathrm{NaCl}+x \mu \mathrm{mol} / \mathrm{L}$ SPS.

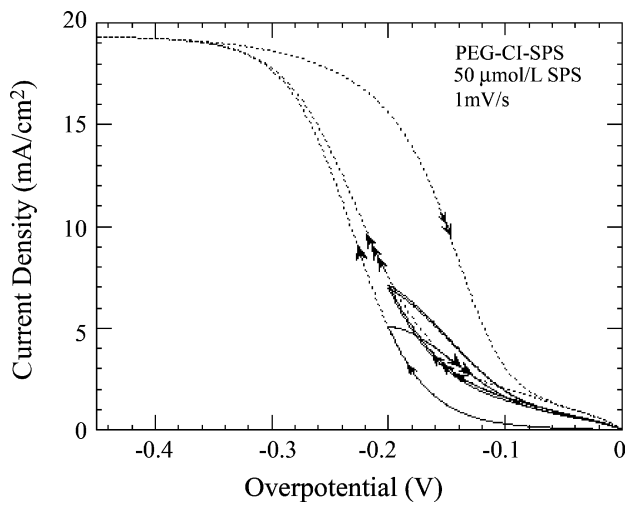

Fig. 2. Variation of the switching potential in multicycle voltammetric experiments reveals the strong effect of potential-dependent adsorption and consumption of the catalyst.

as adsorbing SPS displaces PEG from the interface. The extent of acceleration on the negative-going sweep scales with the SPS concentration, and a strong hysteretic response is evident on the return or positive-going scan. Though not evident in Fig. 1, for the $1 \mathrm{mV} / \mathrm{s}$ sweep rate and $-0.45 \mathrm{~V}$ switching potential, the acceleration attained on the return sweep saturates for SPS concentration greater than $7 \mu \mathrm{mol} / \mathrm{L}$ SPS. Following voltammetry the mechanical polished electrode surface is smooth and bright, indicating that a change of surface chemistry rather than electrode area is responsible for the hysteretic behavior.

Multicycle voltammetry provides a clear demonstration of the potential dependence of SPS adsorption and its consumption. As shown in Fig. 2, for an electrolyte with $50 \mu \mathrm{mol} / \mathrm{L} \mathrm{SPS}$ the second and subsequent negative going sweeps do not follow the path of the preceding return sweep. This is due to significant SPS deactivation at lower overpotentials; as noted earlier, deactivation of adsorbed SPS catalyst occurs most rapidly near $-0.1 \mathrm{~V}$ according to studies of the deactivation of SPS derivatized electrodes [2,6]. A steady-state $\eta-i$ response is generally attained by completion of the second cycle although the precise number of cycles depends on other experimental parameters including the SPS concentration, switching potential and scan rate. The impact of the potential dependence of the SPS adsorption rate constant is revealed by changing the switching potential while the sweep rate and total scan time (2400 s) are held constant. The high metal deposition rate that characterizes the return sweep for a switching potential of $-0.45 \mathrm{~V}$ is not accessible if a value less than $-0.2 \mathrm{~V}$ is used. The kinetics of displacement of the inhibiting PEG-Cl layer by SPS adsorption defined in Eq. (4) is an increasing function of overpotential; voltammetric cycling to larger overpotentials therefore permits a larger increase in the catalyst surface coverage that manifests as higher currents on the return sweep. Multicycle simulations for $6.4 \mu \mathrm{mol} / \mathrm{L}$ SPS and a range of switching potentials are in excellent agreement with experiment [2]. Likewise, ex situ surface analytical measurements, specifically X-ray photoelectron spectroscopy, assaying the surface coverage of SPS or its derivatives reveal a hysteretic potential dependence that is consistent with a monotonic relationship between metal deposition rate and the SPS-derived accelerator surface coverage [39]. 

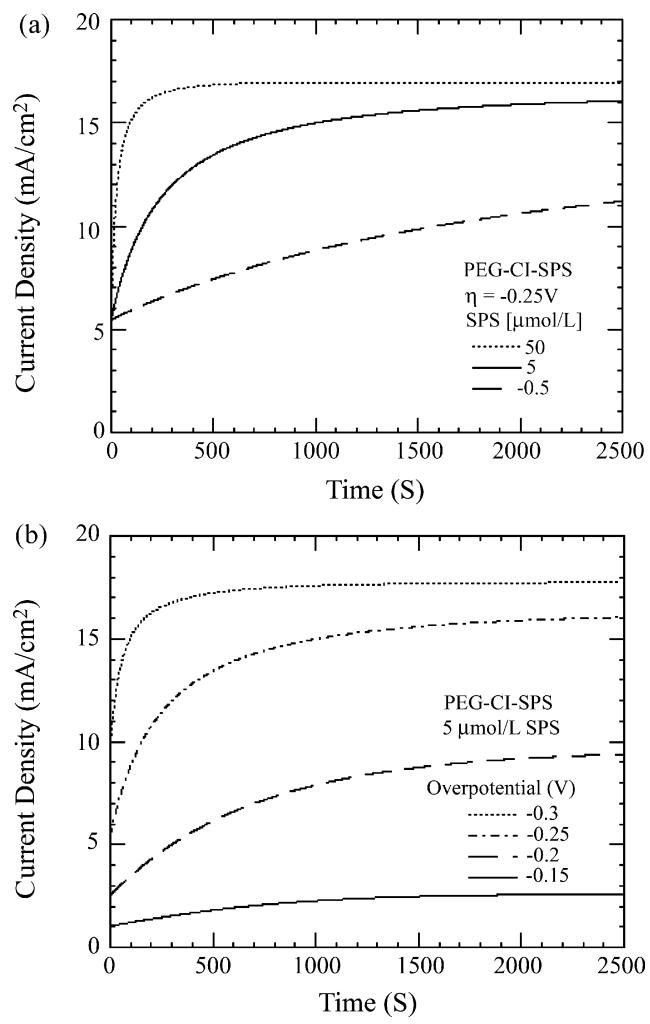

Fig. 3. Rising chronoamperometric transients characterizing the activation of PEG-Cl inhibited electrodes induced by SPS adsorption on stationary copper electrodes: (a) as a function of SPS concentration at $-0.25 \mathrm{~V}$ and (b) as a function of overpotential for $5 \mu \mathrm{mol} / \mathrm{L}$ SPS.

During potentiostatic deposition, such as was used for feature filling in published CEAC-based modeling and experimental studies, a rising current transient is observed [2,6,8]; the gradually increasing current reflects the progressive displacement of the blocking PEG layer caused by SPS adsorption. This behavior, which can be loosely thought of as transiting the $\eta-i$ hysteresis loop at a fixed potential, is captured in the simulations in Fig. 3a and $\mathrm{b}$. For an applied potential of $-0.25 \mathrm{~V}$ the time constant of the transients scales with SPS concentration (Fig. 3a). The potential dependent nature of the SPS adsorption rate is evident in Fig. $3 \mathrm{~b}$ although the effect is convoluted with the increasing significance of $\mathrm{Cu}^{2+}$ transport to the interface at more negative potentials.

In many practical applications of electrodeposition, film growth is accomplished at fixed current rather than potential [20]. In the PEG-Cl-SPS system, deposition under such conditions corresponds approximately to transiting the $\eta-i$ hysteresis loop at a fixed current. Examples of simulated depolarization curves are shown in Fig. $4 \mathrm{a}$ and b. As in the fixed potential experiments, the transient arises from displacement of the rateinhibiting PEG layer by the accumulating SPS. The transient results in a potential excursion at early times that is substantially more negative than that associated with the final steady-state conditions. The time constant for this process scales with the SPS concentration as indicated in Fig. 4a. The initial polarization transient from $0 \mathrm{~V}$ associated with establishing the steady-state $\mathrm{Cu}^{2+}$ concentration field is not treated in these simulations as
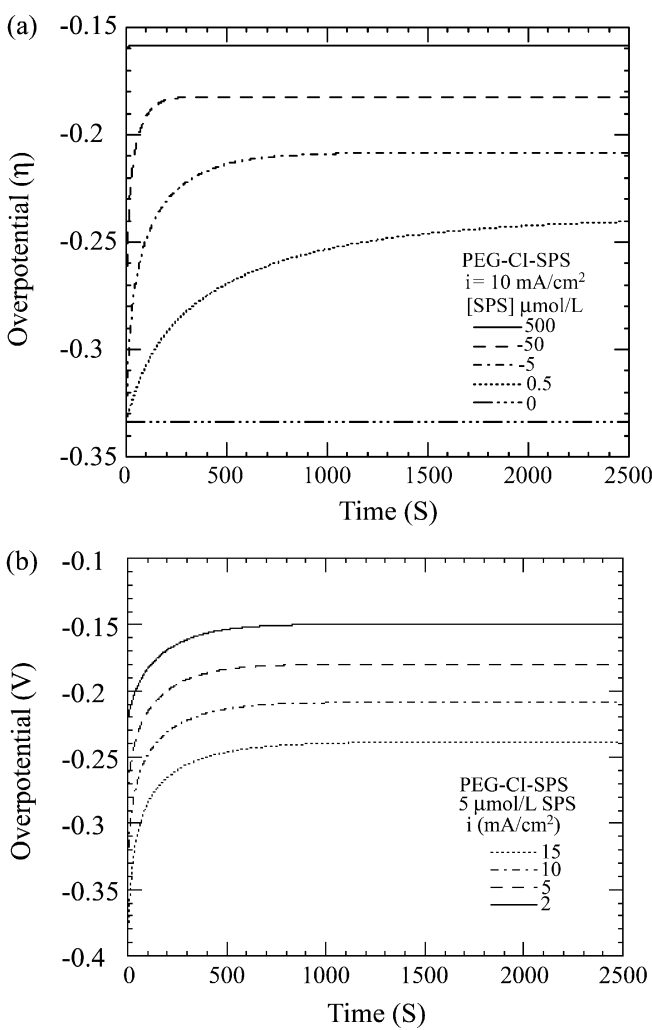

Fig. 4. Depolarizing chronopotentiometric transients characterizing the activation of PEG-Cl inhibited electrodes induced by potential dependent SPS adsorption on stationary copper electrodes: (a) as a function of SPS concentration for an applied current density $(i)$ of $10 \mathrm{~mA} / \mathrm{cm}^{2}$ and (b) as a function of applied current density at $5 \mu \mathrm{mol} / \mathrm{L}$ SPS.

the steady-state boundary layer was assumed to be instantaneously establish and maintained throughout the deposition (i.e., pseudo-steady state diffusion field).

In contrast to the clear effect of accelerator concentration, potential transients obtained at different applied currents, for a fixed SPS concentration of $5 \mu \mathrm{mol} / \mathrm{L}$, exhibit only minor variation of the relaxation time (Fig. $4 \mathrm{~b}$ ). This stands in contrast to the more significant impact on the relaxation time predicted at different potentials in Fig. 3b; the difference can be ascribed to the potential dependence of SPS adsorption. Importantly, unlike individual current-transients, the potential transients and cyclic-voltammetry convolve the potential dependence of the adsorption process with the impact of its accumulation on the metal deposition rate. On the other hand, chronopotentiometry allows the effect of the additives to be assayed under conditions of fixed growth velocity (and specific hydrodynamics), providing a useful counterpoint to the other methods.

\subsection{PEG-Cl-SPS-LEV system}

The ability of a leveler to quench SPS-derived activity is readily examined using the same electroanalytical methods. A brief survey of the literature indicates that levelers are utilized over a broad concentrations ranging between 0.5 and $500 \mu \mathrm{mol} / \mathrm{L}[11,12,26-28,38,40-43]$. Recent experiments have identified two effective levelers for use in damascene process- 


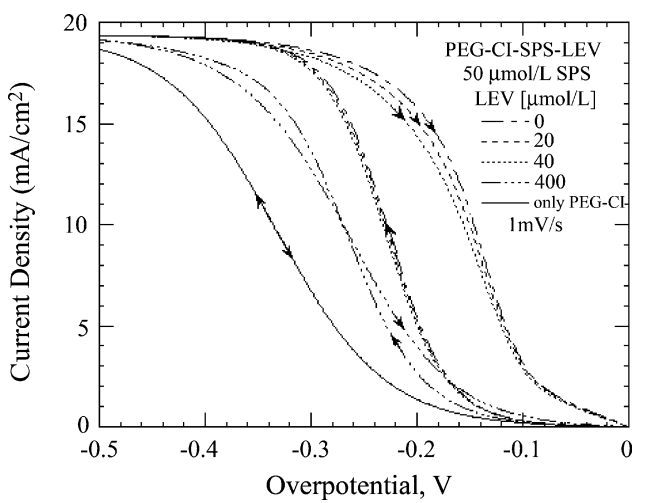

Fig. 5. Slow scan voltammetry reveals the influence of leveler additions on copper deposition rate in an electrolyte comprised of $1.8 \mathrm{~mol} / \mathrm{L} \mathrm{H}_{2} \mathrm{SO}_{4}+0.25 \mathrm{~mol} / \mathrm{L}$ $\mathrm{CuSO}_{4}+88 \mu \mathrm{mol} / \mathrm{L} \mathrm{PEG}+1 \mathrm{mmol} / \mathrm{L} \mathrm{NaCl}+50 \mu \mathrm{mol} / \mathrm{L} \mathrm{SPS}+x \mu \mathrm{mol} / \mathrm{L} \mathrm{LEV}$. For reference, copper deposition in a PEG-Cl (i.e. SPS and LEV free) electrolyte is also shown. Scan rate is $1 \mathrm{mV} / \mathrm{s}$.

ing; $\left(\mathrm{CH}_{3}\right)_{3} \mathrm{C}_{12} \mathrm{H}_{25} \mathrm{~N}^{+} \mathrm{Cl}^{-}$(DTAC) [28] and polyethyleneimine (PEI) [38]. Likewise, two recent publications identify DTAB $[27,44]$ as a leveler; it should, however, be noted that DTAB corresponds to the addition of two additives, $\mathrm{DTA}^{+}$and $\mathrm{Br}^{-}$, where the latter alone is known to significantly perturb PEG and SPS adsorption, potentially complicating evaluation of any interactions that might be attributable to $\mathrm{DTA}^{+}$in that system. In the present case, preliminary $\eta-i$ experiments in the SPSCl-PEG-DTAC system were used to constrain the choice of the adsorption and deactivation kinetics of the leveler [28]. Analysis indicates that the kinetics of DTAC adsorption are relatively slow. Kinetic parameters consistent with this observation, as contained in Table 1, have been used in what follows. If the leveler deactivation process is interpreted as a simple desorption step then the ratio $k_{\mathrm{LEV}}^{+} / k_{\mathrm{LEV}}^{-}$corresponds to the Langmuir adsorption constant, $K$, the reciprocal of which, for the values included in Table 1 , is $\approx 28 \mu \mathrm{mol} / \mathrm{L}$.

Simulations were performed as function of leveler concentration while all other parameters were fixed $\left(C_{\text {SPS }}=50 \mu \mathrm{mol} / \mathrm{L}\right)$. A sampling of the predicted voltammetric behavior of the PEG$\mathrm{Cl}-\mathrm{SPS}-\mathrm{LEV}$ system is given in Fig. 5. The hysteretic curve for the leveler-free PEG-Cl-SPS electrolyte, discussed earlier, is shown as a point of reference along with the voltammetric curve for the fully inhibited PEG-Cl system. Addition of the leveler leads to a decrease in the hysteretic response as well as a shift towards more negative potentials that indicates quenching of the SPS activity. The intersection of the forward and reverse sweep for the electrolytes with higher concentrations of leveler, e.g. $400 \mu \mathrm{mol} / \mathrm{L}$, reflects the kinetic dampening associated with leveler adsorption; these crossing points would not exist for fully equilibrated Langmuir adsorption of the leveler.

Simulations were done at $-0.25 \mathrm{~V}$ as this corresponds to an effective value for feature superfilling in the SPS-Cl-PEG system. The maxima in the transients for the higher leveler concentrations in Fig. 6 arise because the leveler accumulates more slowly due to kinetic limitations on its adsorption. Thus, the initial rise in the current is associated with displacement of PEG by SPS adsorption; the subsequent fall is associated with deactivation of the SPS activity by LEV adsorption through

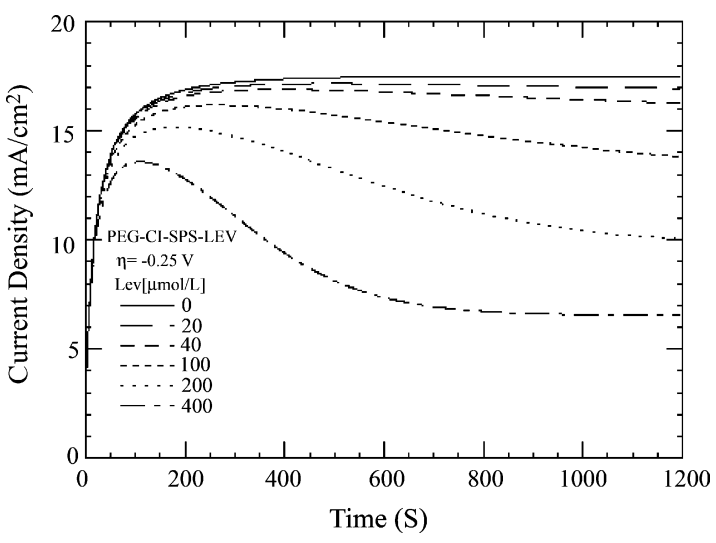

Fig. 6. The influence of leveler concentration on potentiostatic $(-0.25 \mathrm{~V})$ $\mathrm{Cu}$ deposition in an electrolyte comprised of $1.8 \mathrm{~mol} / \mathrm{L} \mathrm{H}_{2} \mathrm{SO}_{4}+0.25 \mathrm{~mol} / \mathrm{L}$ $\mathrm{CuSO}_{4}+88 \mu \mathrm{mol} / \mathrm{L} \mathrm{PEG}+1 \mathrm{mmol} / \mathrm{L} \mathrm{NaCl}+50 \mu \mathrm{mol} / \mathrm{L} \mathrm{SPS}+x \mu \mathrm{mol} / \mathrm{L} \mathrm{LEV}$. Note, for leveler concentrations less than $\sim 40 \mu \mathrm{mol} / \mathrm{L}$ a relatively small perturbation of the metal deposition kinetics is apparent during the first $100 \mathrm{~s}$, a timescale that is longer than typical feature filling times.

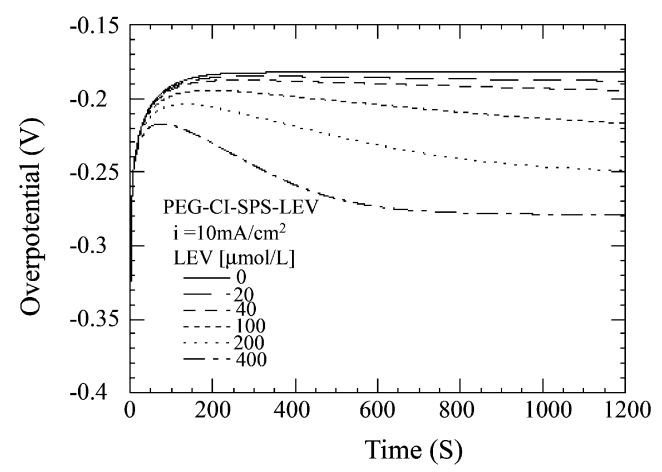

Fig. 7. The influence of leveler concentration during galvanostatic $\left(i=10 \mathrm{~mA} / \mathrm{cm}^{2}\right) \mathrm{Cu}$ deposition in $1.8 \mathrm{~mol} / \mathrm{L} \mathrm{H}_{2} \mathrm{SO}_{4}+0.25 \mathrm{~mol} / \mathrm{L} \mathrm{CuSO}_{4}+$ $88 \mu \mathrm{mol} / \mathrm{L} \mathrm{PEG}+1 \mathrm{mmol} / \mathrm{L} \mathrm{NaCl}+50 \mu \mathrm{mol} / \mathrm{L} \mathrm{SPS}+x \mu \mathrm{mol} / \mathrm{L} \mathrm{LEV}$. Note, for leveler concentrations less than $\sim 40 \mu \mathrm{mol} / \mathrm{L}$ a minor perturbation of the metal deposition kinetics is apparent during the first 100, a timescale that is longer than typical feature filling times.

the leveler-accelerator deactivation term, $\theta_{\mathrm{SPS}} k_{\mathrm{LEV}}^{+} C_{\mathrm{LEV}}^{i}$, in Eq. (2). Significantly, for leveler concentrations below $\sim 50 \mu \mathrm{mol} / \mathrm{L}$, there is negligible impact on the metal deposition rate during the first $\approx 100 \mathrm{~s}$, a time scale that corresponds to sub-micrometer feature filling experiments. This is because $i_{\mathrm{LEV}}^{0}$ and $i_{\mathrm{PEG}}^{0}$ are approximately equal and much less than $i_{\text {SPS }}^{0}$ so that leveler accumulation only affects the current density when its adsorption begins to substantially impact $\theta_{\text {SPS }}$ evolution. In a related fashion, leveler accumulation leads to a minimum in depolarization during galvanostatic deposition as shown in Fig. 7.

\section{Shape change simulations}

The competitive adsorption kinetics derived from measurements on planar electrodes and utilized in the previous simulations of deposition on planar substrates, was also used in combination with the CEAC formalism to explore shape evolution during electrodeposition in submicrometer trenches. For these simulations the initial values of $\theta_{\text {SPS }}$ and $\theta_{\text {LEV }}$ were both 

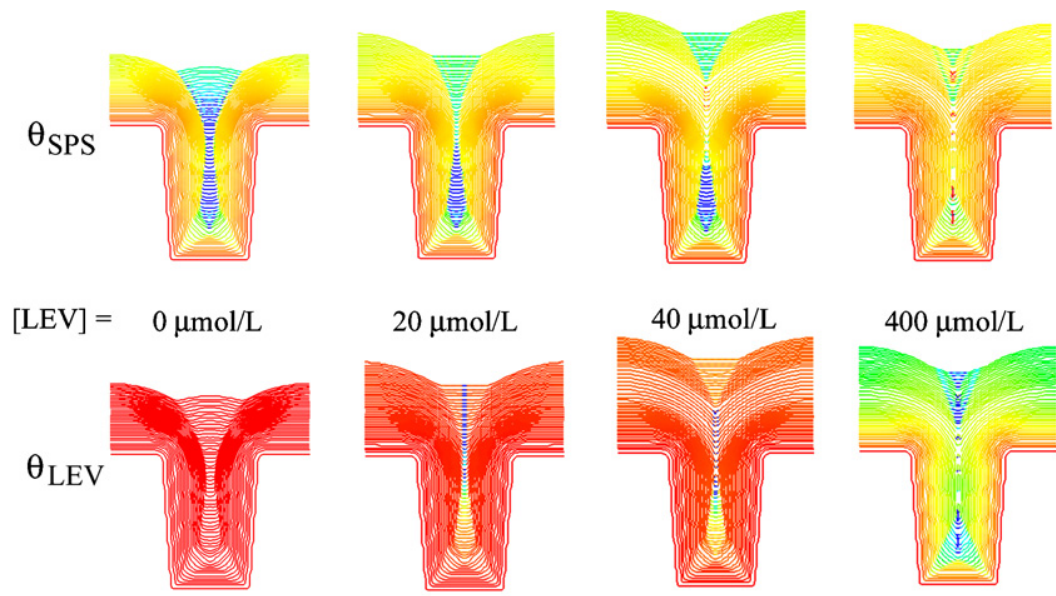

$20 \mu \mathrm{mol} / \mathrm{L}$
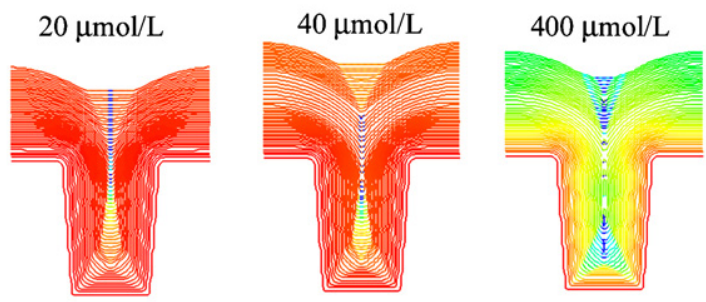

0.00

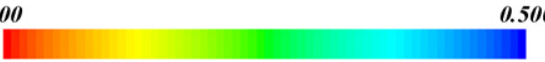

Fig. 8. Trench filling contours for copper electrodeposition at $-0.25 \mathrm{~V}$ in a $1.8 \mathrm{~mol} / \mathrm{L} \mathrm{H}_{2} \mathrm{SO}_{4}+0.25 \mathrm{~mol} / \mathrm{L} \mathrm{CuSO}+88 \mu \mathrm{mol} / \mathrm{L} \mathrm{PEG}+1 \mathrm{mmol} / \mathrm{L} \mathrm{NaCl}+50 \mu \mathrm{mol} / \mathrm{L}$ SPS $+x \mu \mathrm{mol} / \mathrm{L} \mathrm{LEV} \mathrm{electrolyte}\left(0,20,40\right.$ and $400 \mu \mathrm{mol} / \mathrm{L}$ as marked). The contours are colorized to indicate the local coverage of $\theta_{\mathrm{SPS}}$ (top row) and $\theta_{\mathrm{LEV}}(\mathrm{bottom}$ row) evolution resulting from the combination of accumulation from the electrolyte and area change as incorporated in the CEAC mechanism.

set to zero and allowed to evolve during $\mathrm{Cu}$ deposition in the SPS-Cl-PEG-(LEV) electrolyte. The simulated feature filling contours are shown in Fig. 8. The simulations were performed using a level set formulation of the CEAC model [13] within a Python framework developed at NIST that is known as FiPy [29]. The growth contours are colorized to reflect local coverages of SPS (top) and LEV (bottom) throughout the deposition process. The simulations shown were performed at an overpotential of $-0.25 \mathrm{~V}$ with $50 \mu \mathrm{mol} / \mathrm{L}$ SPS and the indicated leveler concentration. The corresponding simulations of currenttransients during deposition on planar substrates are shown in Figs. $3 \mathrm{a}$ and 6.

In the case of deposition in the PEG-Cl-SPS system, enrichment of SPS accelerator on concave sections as well as the resulting void-free bottom-up filling and overfill bump are all evident in Fig. 8. Geometry driven enrichment of the accelerator coverage occurs initially at the bottom corners as they have the highest curvature and thus area-driven enhancement of coverage (the curvature $\kappa$ in Eq. (2) is in fact infinite for sharp corners). When the growth fronts from these corners impinge to form a V-notch, further accelerator enrichment occurs at the resulting corner. The resulting local acceleration leads to a new growth front that, initially forming a flat bottom surface, accelerates upward as its SPS coverage is steadily enriched by transfer of adsorbed SPS from the sidewall area that is being eliminated. This bottom-up filling continues until this growth surface reaches the top of the feature (or the sidewalls meet above it in the case of failure, not shown here) at which point an inversion of curvature occurs as the growth front expands beyond the original free surface. The continued enhancement of deposition on this surface is a legacy of the enhanced SPS coverage and leads to the undesired bump above the feature.

It is worth noting that the distinct shape transitions predicted to accompany the bottom-up growth dynamic have been observed experimentally for a wide range of conditions and chemical system [13-16,18,19,25,41,45-49]. Furthermore,
CEAC-based shape-change simulations and corresponding trench and via filling experiments in PEG-Cl-SPS containing electrolytes show excellent agreement with many aspects of the time-dependent filling [2]. Likewise, fundamental experimental trends with accelerator concentration (initial surface coverage in the case of SPS derivatized electrodes) are captured by CEACbased feature filling simulations [2,13-15].

So-called "leveling" additives are added to the superfilling PEG-Cl-SPS electrolytes in an attempt to control the overshoot phenomena and associated bump formation above the filled features $[27,28,40,41,49]$. The impact of including leveler in the simulations becomes increasingly apparent both in SPS coverage and the associated geometry of filling as the concentration of leveler in the PEG-Cl-SPS electrolyte increases from 20 to $400 \mu \mathrm{mol} / \mathrm{L}$. For $20 \mu \mathrm{mol} / \mathrm{L}$ leveler, enrichment of adsorbed leveler due to area reduction is evident as the bottom surface approaches the top of the feature; however, some enhancement of SPS coverage at the same locations has still occurred, indicating that the LEV coverage was not high enough to entirely force the deactivation of SPS. Nonetheless, comparison of the surface coverages of the accelerator and leveler above the trench with those in the neighboring field, derived solely from electrolyte adsorption, are testament to the overwhelming importance of the area change term in Eqs. (2) and (3). The low coverage of LEV in the field is consistent with prediction of insignificant impact of leveler accumulation for deposition on planar substrates during the $\approx 100 \mathrm{~s}$ time scale of the feature filling (see Fig. 6). Nonetheless, the localized area reduction accompanying bottom-up filling has resulted in substantial enrichment of the adsorbed leveler by the time that the bottom-up growth front approaches the top of the feature. Significantly, while the bottom-up filling dynamic is still evident, the leveler accumulation on the bottom-surface due to area change is such that bump formation does not occur.

Doubling the leveler concentration to $40 \mu \mathrm{mol} / \mathrm{L}$ results in sufficient buildup of the leveler coverage to completely nul- 
lify the SPS enrichment of the bottom-up growth front as it approaches the top of the feature. Discontinuities in the colorized leveler contours along the centerline at the top of the feature also indicate the formation of tiny voids.

Increasing the leveler concentration an order of magnitude to $400 \mu \mathrm{mol} / \mathrm{L}$ results in extensive leveler adsorption from the electrolyte that, when enhanced by area reduction within the filling feature, completely displaces all adsorbed SPS (note the red regions along midline of SPS coverage plot). As a result it eliminates the bottom-up superfilling dynamic. Impingement of the sidewalls forms voids that are again visible as discontinuities in the colorized contours along the feature centerline.

Comparison between the CEAC simulations and experiments, both electroanalytical and feature filling, has been very favorable to date [2,13-15]. In the case of copper plating, enrichment of SPS adsorbate coverage by area change is key to the bottom-up feature filling. The inverse, namely SPS deactivation by area-change driven enrichment of a leveler, is central to controlling undesired overfilling of damascene structures [2,26]. The CEAC construct has also been applied to other chemically distinct electrodeposition systems, such as silver [23] and gold [24], as well as chemical vapor deposition of copper [25]. For all these systems, the impact of area change within filling features on the evolution of adsorbate coverage and thus metal deposition rate has been shown to be central to understanding and controlling superfill of fine features.

\section{Conclusions}

The quantitative connection between additive effects on metal electrodeposition on planar electrodes and its connection with area change effects during growth on non-planar surfaces is detailed in terms of the CEAC mechanism. The central importance of additive enrichment and dilution effects that accompany electrode area change is demonstrated within the context of $\mathrm{Cu}$ electrodeposition and the filling of sub-micrometer features in damascene processing. The area change driven brightening and leveling mechanism is completely distinct from the long established diffusion-adsorption-consumption model of leveling. The CEAC formalism provides a means of extracting kinetic parameters from electroanalytical measurements for use in shape change algorithms that quantitatively predict feature filling on non-planar substrates. The simulation tools may also be used in combination with experiments to identify and optimize electrolytes for a given application.

\section{References}

[1] P.C. Andricacos, C. Uzoh, J.O. Dukovic, J. Horkans, H. Deligianni, IBM J. Res. Dev. 42 (1998) 567.

[2] T.P. Moffat, D. Wheeler, M. Edelstein, D. Josell, IBM J. Res. Dev. 49 (2005) 19.

[3] W.-P. Dow, M.-Y. Yen, W.-B. Lin, S.-W. Ho, J. Electrochem. Soc. 152 (2005) C769.

[4] S.K. Cho, S.K. Kim, J.J. Kim, J. Electrochem. Soc. 152 (2005) C330.

[5] P.M. Vereecken, R.A. Binsted, H. Deligianni, P.C. Andricacos, IBM J. Res. Dev. 42 (2005) 3.

[6] T.P. Moffat, D. Wheeler, D. Josell, J. Electrochem. Soc. 151 (2004) C262.
[7] B.M. Basol, J. Electrochem. Soc. 151 (2004) C765.

[8] M. Tan, J. Harb, J. Electrochem. Soc. 150 (2003) C420.

[9] W.P. Dow, M.Y. Yen, Electrochem. Solid State Lett. 8 (2005) C161.

[10] T.P. Moffat, D. Wheeler, C. Witt, D. Josell, Electrochem. Solid State Lett. 5 (2002) C110.

[11] Y. Cao, P. Taephaisitphongse, R. Chalupa, A. West, J. Electrochem. Soc. 148 (2001) C466.

[12] P. Taephaisitphongse, Y. Cao, A.C. West, 148 (2001) C492.

[13] D. Wheeler, D. Josell, T.P. Moffat, J. Electrochem. Soc. 150 (2003) C302.

[14] D. Josell, D. Wheeler, W.H. Huber, J.E. Bonevich, T.P. Moffat, J. Electrochem. Soc. 148 (2001) C767.

[15] T.P. Moffat, D. Wheeler, D. Josell, Electrochem. Solid State Lett. 4 (2001) C26.

[16] A.C. West, S.T. Mayer, J. Reid, Electrochem. Solid State Lett. 4 (2001) C50.

[17] J.J. Kelly, A.C. West, Electrochem. Solid-State Lett. 2 (1999) 561.

[18] J. Reid, Jpn. J. Appl. Phys., Part 140 (2001) 2650

[19] T.P. Moffat, J.E. Bonevich, W.H. Huber, A. Stanishevsky, D.R. Kelly, G.R. Stafford, D. Josell, J. Electrochem. Soc. 147 (2000) 4524.

[20] J. Reid, C. Glack, S.J. Hearne, Electrochem. Solid State Lett. 6 (2003) C26.

[21] D. Wheeler, T.P. Moffat, G.B. McFadden, S. Coriell, D. Josell, J. Electrochem. Soc. 151 (2004) C538.

[22] G. McFadden, S. Coriell, T.P. Moffat, D. Josell, D. Wheeler, W. Schwarzacher, J. Mallett, J. Electrochem. Soc. 150 (2003) C591.

[23] B.C. Baker, M. Freeman, B. Melnick, D. Wheeler, D. Josell, T.P. Moffat, J. Electrochem. Soc. 150 (2003) C61.

[24] D. Josell, D. Wheeler, T.P. Moffat, J. Electrochem. Soc. 153 (2006) C11.

[25] D. Josell, S. Kim, D. Wheeler, T.P. Moffat, S.G. Pyo, J. Electrochem. Soc. 150 (2003) C368.

[26] T.P. Moffat, D. Wheeler, S.-K. Kim, D. Josell, J. Electrochem. Soc. 153 (2006) C127.

[27] J. Reid, E. Webb, J. Sukamto, Y. Takada, T. Archer, in: H. Deligianni, S.T. Mayer, T.P. Moffat, G.R. Stafford (Eds.), Electrochemical Processing in ULSI and MEMS, The Electrochemical Society Inc., 2005, p. 184.

[28] S.K. Kim, D. Wheeler, D. Josell, T.P. Moffat, J. Electrochem. Soc. 153 (2006) C826.

[29] D. Wheeler, J. Guyer, J.A. Warren, FiPy: A Finite Volume PDE Solver Using Python, http://www.ctcms.ist.gov/fipy/.

[30] C. Witt, J. Srinivasan, R. Carpio, in: H. Deligianni, S.T. Mayer, T.P. Moffat, G.R. Stafford (Eds.), Electrochemical Processing in ULSI and MEMS, The Electrochemical Society, Inc., 2005, p. 57.

[31] C. Madore, M. Matlosz, D. Landolt, J. Electrochem. Soc. 143 (1996) 3927.

[32] C. Madore, D. Landolt, J. Electrochem. Soc. 143 (1996) 3936

[33] A.C. West, J. Electrochem. Soc. 147 (2000) 227.

[34] R. Chalupa, Y. Cao, A.C. West, J. Appl. Electrochem. 32 (2002) 135.

[35] J. Dukovic, in: H. Gerischer, C.W. Tobias (Eds.), Advances in Electrochemical Science and Engineering, vol. 3, 1994, p. 117.

[36] S.I. Krichmar, Elektrokhimiya 1 (1965) 858.

[37] D. Roha, U. Landau, J. Electrochem. Soc. 137 (1990) 824.

[38] S.K. Kim, D. Josell, T.P. Moffat, J. Electrochem. Soc. 153 (2006) C616.

[39] T.P. Moffat, P.J. Chen, A. Castillo, B. Baker, W.F. Egelhoff, L. Richter, J. Electrochem. Soc. (submitted for publication).

[40] M. Hasegawa, Y. Negishi, T. Nakanishi, T. Osaka, J. Electrochem. Soc. 152 (2005) C221.

[41] S.-K. Kim, S.K. Cho, J.J. Kim, Leveling of superfilled damascene Cu film, Electrochem. Solid State Lett. 9 (2006) C25.

[42] M. Wuensche, W. Dahms, H. Mayer, R. Schumacher, Electrochim. Acta 39 (1994) 1133.

[43] W. Plieth, Electrochem. Acta 37 (1992) 2115.

[44] E.D. Eliadis, R.C. Alkire, J. Electrochem. Soc. 145 (1998) 1218.

[45] Q.-T. Jiang, A. Frank, B. Carpenter, R. Carpio, T. Ritzdorf, R.A. Auger, K. Pfeifer, J.D. Luttmer, in: A.J. mCKarrow, Y. Shacham-Diamand (Eds.), Advanced Metallization Conference 2001, MRS, Warrendale, PA, 2001, p. 215;

A. Frank, Q.T. Jiang, L. Chen, B. Carpenter, R. Carpio, T. Ritzdorf, K. Pfeifer, in: D. Edelstein, G. Dixit, Y. Yasuda, T. Ohba (Eds.), Advanced Metallization Conference, MRS, Warrendale, PA, 2000, p. 181. 
[46] Y.H. Im, M.O. Bloomfield, S. Sen, T.S. Cale, Elechem. Solid-State Lett. 6 (2003) C42.

[47] B. Zheng, R. He, B. Mikkola, J. Wang, C. Long, C. Yu, Z.-W. Sun, E. Step, J. Chen, R. Emamai, Z.A. Wang, R. Nayak, T. Taylor, G. Dixit, in: A.J. McKerrow, Y. Shacham-Diamand, S. Zaima, T. Ohba (Eds.), Advanced Metallization Conference 2001, MRS, Warrendale, PA, 2001, p. 197.
[48] T. Haba, T. Itabashi, H. Akahoshi, H. Miyazaki, in: B.M. Melnick, T.S. Cale, S. Zaima, T. Ohta (Eds.), Advanced Metallization Conference 2002, MRS, Warrendale, PA, 2003, p. 361

[49] M.X. Yang, D.X. Mao, C.M. Yu, J. Dukovic, M. Xi, Solid State Technol. 46 (2003) 37 